$\mathbb{T}$ periodica polytechnica

Transportation Engineering

41/2 (2013) 133 137

doi: 10.3311/PPtr.7114

http://periodicapolytechnica.org/tr

Creative Commons Attribution (1)

RESEARCH ARTICLE

\section{Carrying Capacity of Horizontal Cylindrical Shells on Saddle Supports}

\author{
Ondřej Voltr / Jan Peňáz
}

Received 2013-07-22

\begin{abstract}
The article is devoted to the influence of carrying capacity on the stability of laterally loaded cylindrical shells. The influence of initial imperfections on the stability of the laterally loaded shell is also taken into consideration. The investigated structure is a simplified variant of the case of cylindrical shell (road tank - for example Paščenko [5]; Paščenko, Stejskal [6]) located on two saddle supports. The initial shape imperfections are created by pushing the saddle support into the shell. The new deformed shape is used as an imperfect shell in the following nonlinear analysis of stability. The main objective of the analysis is to find the reduction factor $\alpha$ depending on the change of geometric parameters of the numerical model (embracing angle $2 \theta$, wall thickness $t$ ). The shell deformation around the saddle support is also monitored. Numerical analysis is carried out by means of a computer program COSMOS/M based on the finite element method (FEM).
\end{abstract}

\section{Keywords}

cylindrical shell · FEM - initial imperfections · loss of stability $\cdot$ saddle support

\section{Acknowledgement}

This work was supported by the University of Pardubice, project No. 51030/20/SG530001.

\section{Ondřej Voltr}

Department of Mechanics, Materials and Machine Parts, Jan Perner Transport Faculty, University of Pardubice, Studentská 95, 53210 Pardubice 2, Czech Republic e-mail: ondrej.voltr@student.upce.cz

\section{Jan Peňáz}

Department of Mechanics, Materials and Machine Parts, Jan Perner Transport Faculty, University of Pardubice, Studentská 95, 53210 Pardubice 2, Czech Republic e-mail: jan.penaz@student.upce.cz

\section{Introduction}

This article is focused on a research concerning the loss of stability of road tanks located on two saddle supports.

A simplified version in the form of a horizontal cylindrical shell transversally loaded by a firmly joined rigid punch (saddle support) is investigated (Voltr, Paščenko [8]). The effect of initial imperfections on stability of the shell is namely examined. In the first step of research, stability analysis is performed only in the elastic area. The models with frequently used variants of embracing angle $2 \theta=60,90,120^{\circ}$ and range of ratio $R / t \in\langle 70 ; 250\rangle$ are used. The $R / t$ is ratio of radius of shell $R$ to the thickness of the shell $t$. The analysis monitors the change of the reduction factor $\alpha$ in response to changes of the shell thickness $t$. Moreover, attention is paid to the nature of deformation of the cylindrical shell during loading.

Additional bending load is introduced into the shell through initial shape imperfections (Volmir [7]). It usually results in a decrease of carrying capacity (Wunderlich, Albertin [9]; Wunderlich, Deml [10|). However, sometime this effect is quite opposite and carrying capacity of imperfect shell grows. In this case the imperfection can have a reinforcing effect. Within this research, the imperfection is created by means of the auxiliary linear analysis when the support is pushed into the shell of a precisely defined value $2 \mathrm{~mm}$. Thus, the deformed model is then used as a starting model for the following nonlinear analysis.

\section{Computational Model}

The thin-walled cylindrical shell with a length $L=300 \mathrm{~mm}$, diameter $D=150 \mathrm{~mm}$ and wall thickness t (thickness varies in the range $0.3 \div 1.1 \mathrm{~mm}$ in increments of $0.1 \mathrm{~mm}$ ) is adopted for the numerical model (Voltr, Paščenko [8]) (see Fig. 1). The shell is provided at both ends with flat circular covers with a thickness $t_{1}=30 \mathrm{~mm}$. This thickness ensures sufficient rigidity of the covers preventing their excessive deformation which could have a negative effect on analyzed results. In the middle span of the shell $\left(L_{1}\right)$, the stiff punch in the shape of a saddle support with a width $b=20 \mathrm{~mm}$ and embracing angle $60^{\circ}, 90^{\circ}, 120^{\circ}$ is firmly connected to the cylindrical shell.

The computational model is simply supported in two bound- 
ary element nodes $A, B$ and further, the saddle support is prevented from lateral displacements and tilting (see Fig. 11). During the loss of stability, the shell may thus exhibit both symmetrical and nonsymmetrical deformation in regards to the vertical plane passing through the axis of the cylindrical shell. The computational model is loaded through the saddle support with a vertical force $F$.

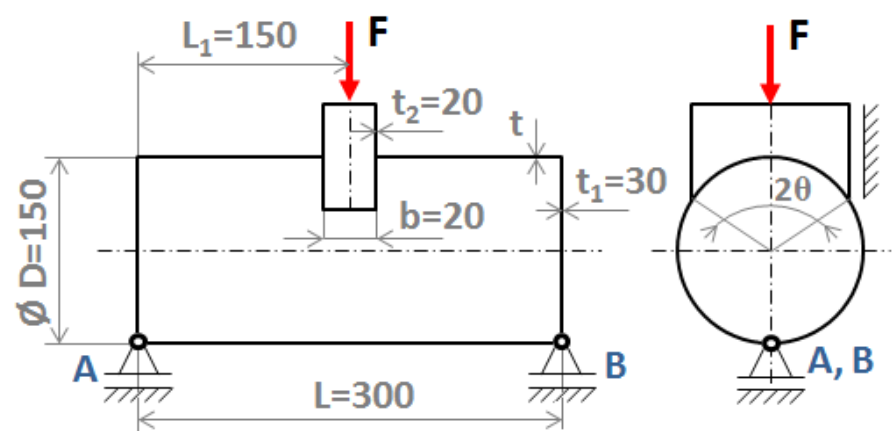

Fig. 1. Geometric parameters of the computational model

The material of all parts of the model (shell, covers, saddle support) has the same mechanical properties, particularly Young modulus of elasticity $E=1.9 \times 10^{5} \mathrm{MPa}$ and Poisson's ratio $\mu=0.3$.

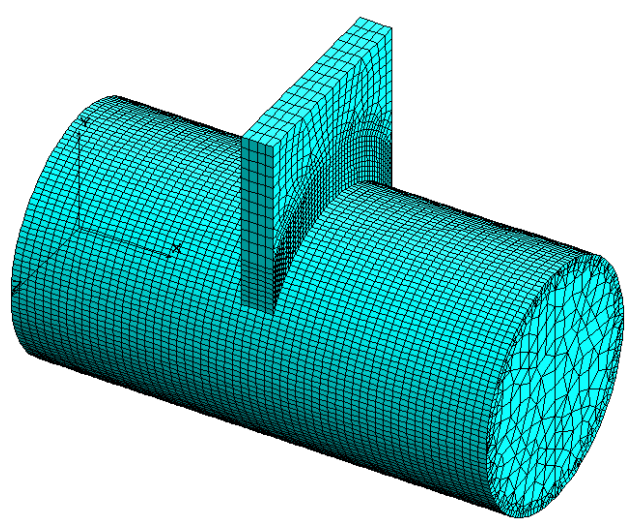

Fig. 2. Initial undeformed model $\left(2 \theta=120^{\circ}\right)$

\section{Result of Evaluation of Numerical Analyses}

As mentioned before, thickness of the shell $t$ varies in the range $0.3 \div 1.1 \mathrm{~mm}$ (in increments of $0.1 \mathrm{~mm}$ ). That means that depending on varying thickness $t$ were performed 9 numerical analyses of the perfect shell (without initial imperfection GNA) and 9 analyses of the imperfect shell (with initial imperfection - GNIA). All of that for each embracing angle $2 \theta=60^{\circ}$, $90^{\circ}, 120^{\circ}$. So that means overall 54 nonlinear numerical analyses were performed.

Reduction factor $\alpha$ is calculated from results of these individual analyses. It represents the influence of initial imperfections on stability of examined shell. Reduction factor is gained as a ratio of results of nonlinear numerical analyses of imperfect shell (with initial imperfection) to the perfect shell (without initial imperfection). Both types of analyses are in the elastic area. These ratios can be seen in Fig. 5, Fig. 8 and Fig. 11 as individual dots of curves of reduction factor $\alpha_{120^{\circ}}, \alpha_{90^{\circ}}, \alpha_{60^{\circ}}$.

The numerical results carried out in this configuration of boundary conditions show that the lateral deformations of the cylindrical shell may be considered for the first natural mode. The shell has a tendency to lay on one edge of the saddle. The second natural mode is then symmetric push of the saddle support into the cylindrical shell. Certain combination of both natural shapes appeared during many analyses. Initially, the symmetrical push of the saddle support into the shell takes place. It corresponds to the linear part of the load curve. Then the lateral displacements appear with a subsequent loss of stability of the cylindrical shell. This corresponds to a nonlinear part of the load curve. Course of the lateral deformation is characterized by redistributing originally symmetrically spaced waves into the shape with a significant buckle and valley around the corner of the saddle on the incline side. Other deformation is almost smoothed (see Fig. 7). Previous text concerned the behavior of the perfect ideal shells without initial imperfections.

Deformation of imperfect shells during loss of stability is influenced by intentionally established initial imperfections. The general nature of loss of stability is similar to the previous case however in addition, some local deformations of various depths and shapes appear in vicinity of the saddle support. For greater shell thickness, these deformations often disappear with increasing load. Sometimes, local valleys of nearly circular shape appear there.

Note: The deformation scale of the following computational models is enlarged for better visibility and understanding of the process of loss of stability.

Note: Presented examples of deformed shells (of various embracing angles) were chosen according to general patterns which were observed on the whole scope of the analyses. Therefore was more important to show two deformed imperfect shells of embracing angle $120^{\circ}$ (two different natural modes, Fig. 6 , Fig. 7 rather than show one perfect and one imperfect shell as for embracing angles $90^{\circ}$ a $60^{\circ}$ (Fig. 9 , Fig. 10, Fig. 12, Fig. 13.

\subsection{Carrying capacity - geometrically nonlinear analysis GNA}

One remarkable nonlinear analysis of shell without initial imperfection (ECCS [2]; Eurocode 3 [3]) is for example shown in the following text. The analysis takes into the consideration only geometrical nonlinearity (large displacements) with material behavior in elastic range (Bushnell [1]; Zienkiewicz [11]). Fig. 3 shows the load (equilibrium) curve for model $t=0.5$ and $2 \theta=120^{\circ}$. Six gradual losses of stability occur during the loading of the shell. Each loss of stability causes decrease of carrying capacity and is accompanied by redistribution of deformation into new shape. This means that the shell gradually goes through various equilibrium states. The shell resists the acting load and the waves and buckles are redistributed during the whole loading. 


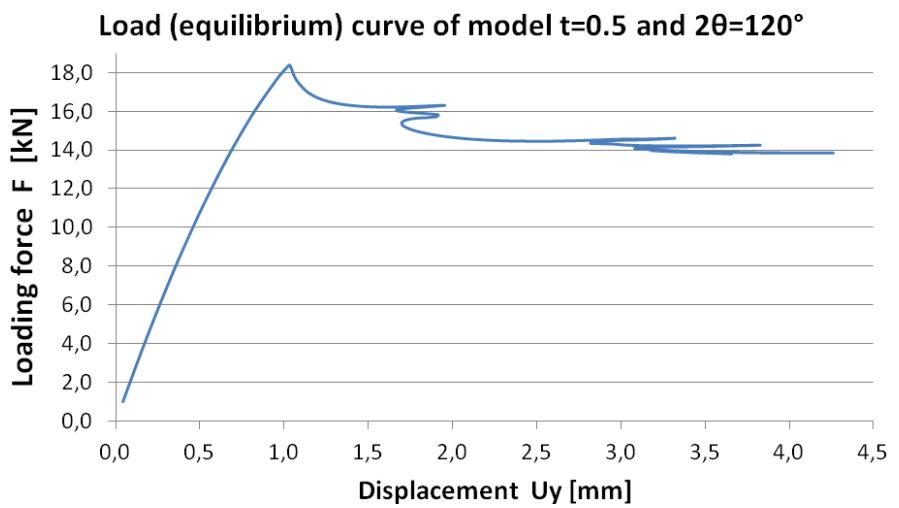

Fig. 3. Example of load (equilibrium) curve

At first, a symmetrical deformation is clearly visible in the saddle area. Then the starting lateral displacements appear with a subsequent first loss of stability $\left(U_{y} \approx 1 \mathrm{~mm}\right.$, see Fig. 33). Lateral displacement of the shell continues till the second loss of stability happens $\left(U_{y} \approx 2 \mathrm{~mm}\right)$. The shell lays on one edge of the saddle on the incline side. The third loss of stability occurs again at displacement $U_{y} \approx 2 \mathrm{~mm}$ and significant circle valley appears in the saddle area. Last three losses of stability occur at range of displacements $U_{y} \approx 3-4 \mathrm{~mm}$. These are accompanied by several circle valleys on the sides of the shell. The valleys are visible in Fig. 4 The final deformed model at the end of loading is also shown in Fig. 4

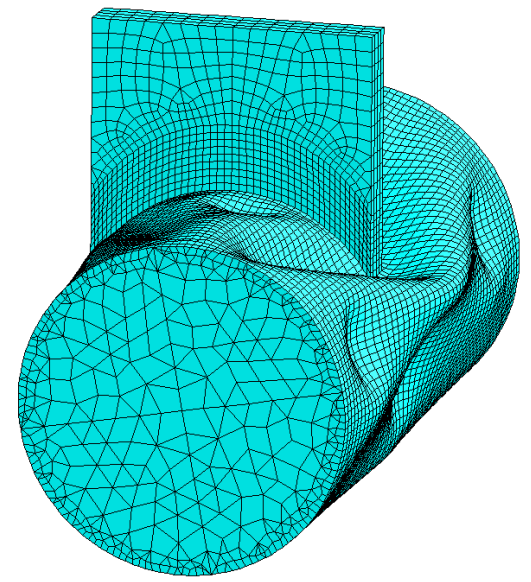

Fig. 4. Deformed perfect model at the end of loading, $t=0.5 \mathrm{~mm}$, $2 \theta=120^{\circ}$

The following text is devoted to the results of the computational analysis of the stability of cylindrical shells on the saddle support for different embracing angles.

\subsection{Embracing angle $120^{\circ}$}

The graph in Fig 5 shows that the first part of the curve of reduction factor $\alpha_{120^{\circ}}$ decreases with increasing parameter $R / t$. Resistance to loss of stability of the structure decreases due to higher sensitivity to initial imperfections. This trend is expected and the curve is approximately equidistant to the curves of reduction factors $\alpha_{x}$ specified in the European recommendations ECCS [2]. There are three fabrication tolerance quality classes: class A (excellent quality), class B (high quality) and class C (normal quality). Class B is considered for normal production. Last two points of the curve $\alpha_{120^{\circ}}$, corresponding to the wall thickness 0.3 and $0.4 \mathrm{~mm}(R / t=250$, respectively 187.5$)$, show certain differences. Slight reinforcement of the structure followed by growth of the observed curves can be at this moment explained by the proximity of the first and the second natural modes (see Fig. 6 and Fig. 77). The shell is forced, due to the significant initial imperfections, to lose its stability in the second mode corresponding to higher stability resistance.

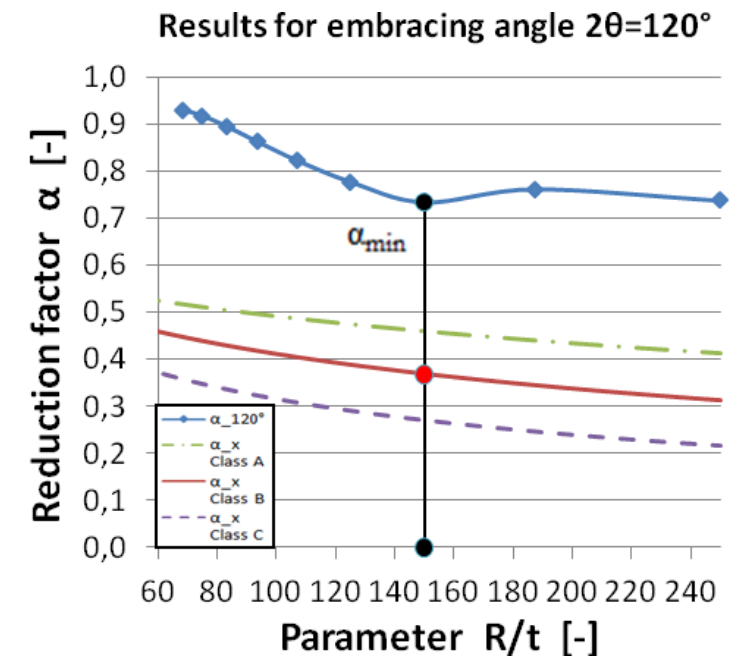

Fig. 5. The dependence of the reduction factor $\alpha$ on the parameter $R / t$, $2 \theta=120^{\circ}$

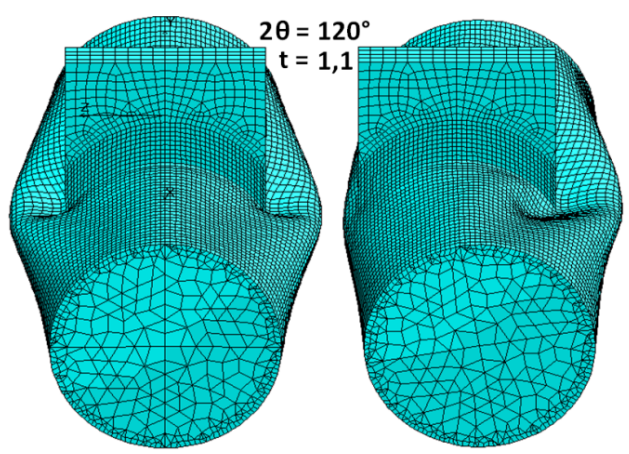

Fig. 6. Typical first natural mode - deformed imperfect model at the beginning (left) and the end of loading (right), $t=1.1 \mathrm{~mm}, 2 \theta=120^{\circ}$

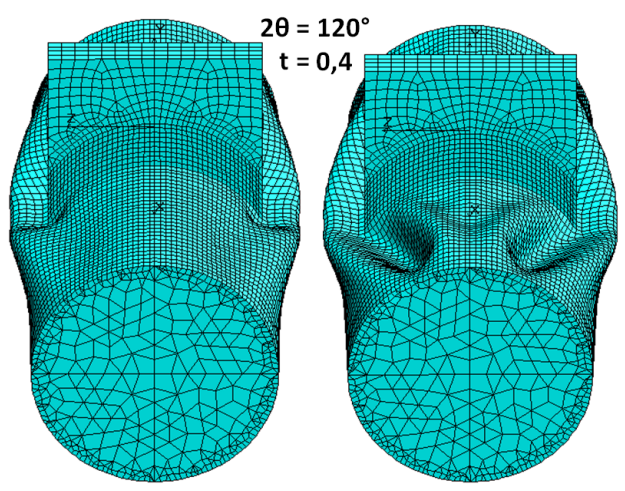

Fig. 7. Typical second natural mode - deformed imperfect model at the beginning (left) and the end of loading (right), $t=0.4 \mathrm{~mm}, 2 \theta=120^{\circ}$ 


\subsection{Embracing angle $90^{\circ}$}

Comparing this case to the previous one $\left(2 \theta=120^{\circ}\right)$, the imperfect models of embracing angle of $90^{\circ}$ show that the curves of reduction factor $\alpha_{90^{\circ}}$ are significantly different from the expected curves in three cases. At the beginning of the curve, for $t=1.1 \mathrm{~mm}$ and $R / t=68.2$, the coefficient $\alpha_{90^{\circ}}$ lies significantly above the value $\alpha=1.0$ (see Fig. 8 ).

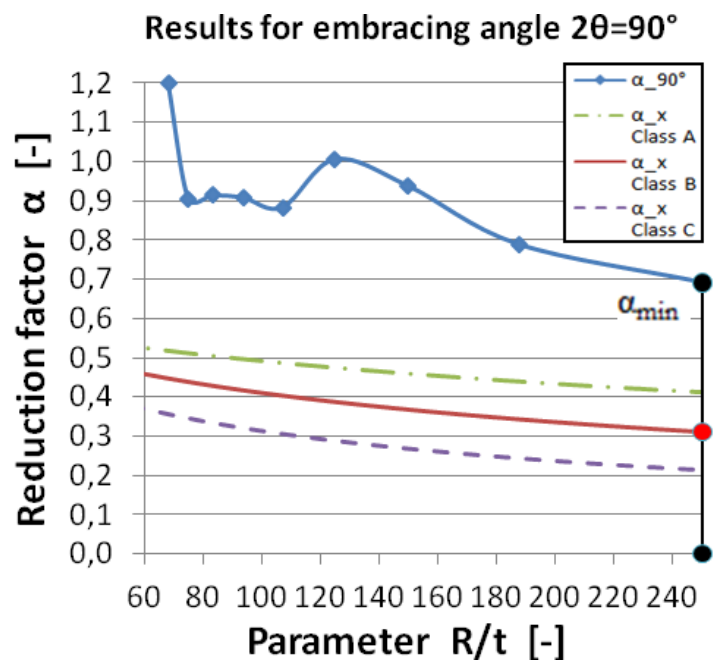

Fig. 8. The dependence of the reduction factor $\alpha$ on the parameter $R / t$, $2 \theta=90^{\circ}$

This is caused by used initial imperfections which result in the formation of other local additional buckles reinforcing the shell. When the increased limit load is achieved then a sudden drop in load due to loss of stability occurs. It is accompanied by a redistribution of the buckles. The shell can thus gradually go through various equilibrium states. Even though the course of deformation of the perfect and imperfect models is quite different, the final shape of the deformation is very similar (lateral displacements, see Fig. 9 and Fig. 10,

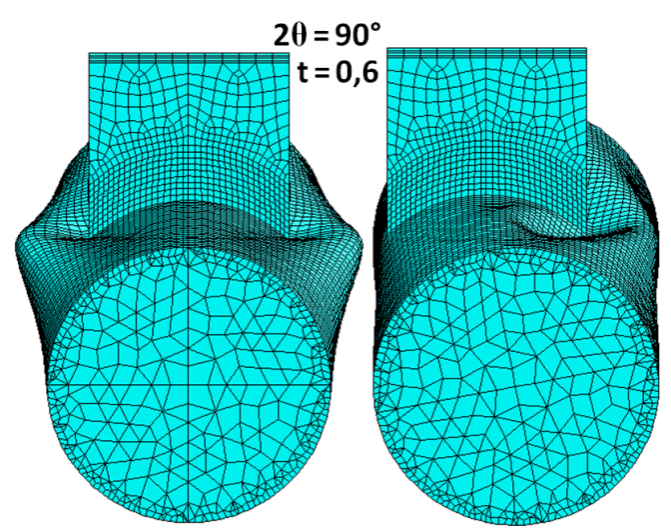

Fig. 9. Deformed perfect model at the beginning (left) and the end of loading (right), $t=0.6 \mathrm{~mm}, 2 \theta=90^{\circ}$

\subsection{Embracing angle $60^{\circ}$}

A typical feature of this embracing angle is a combination of symmetric and asymmetric deformation. From the beginning, the saddle support is pushed into the cylindrical shell symmetrically. This is followed by lateral deformation and pushing the

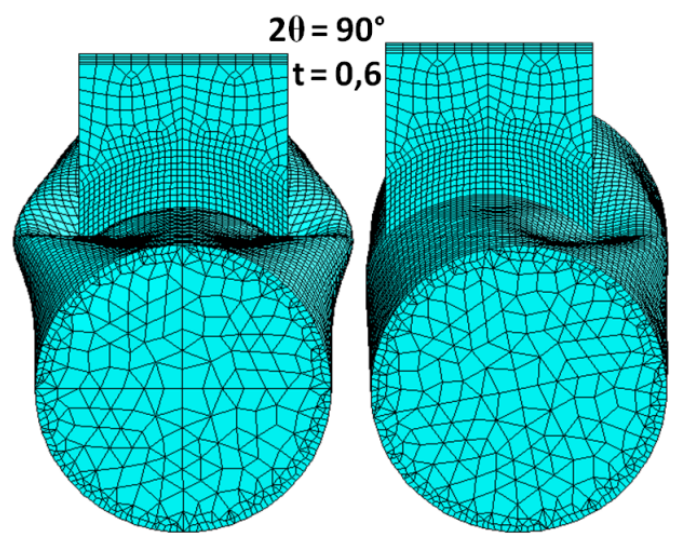

Fig. 10. Deformed imperfect model at the beginning (left) and the end of loading (right), $t=0.6 \mathrm{~mm}, 2 \theta=90^{\circ}$

shell on one edge of the saddle support. Reduced carrying capacity (in terms of limit load) is caused by a small embracing angle - saddle support is narrow and therefore sensitive to early buckling and snap-through into the shell. However, the saddle support with small embracing angle in combination with the initial imperfections results in a significant reinforcement of the structure practically within the entire considered span of parameter $R / t$ (see Fig. 11). Reduction factor is significantly higher than would be expected from the models with embracing angles of $90^{\circ}$ and $120^{\circ}$.

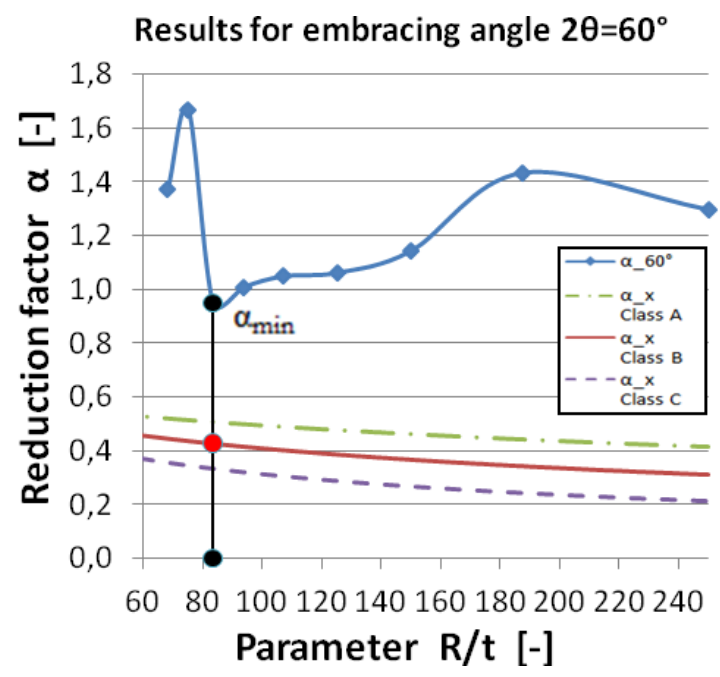

Fig. 11. The dependence of the reduction factor $\alpha$ on the parameter $R / t$, $2 \theta=60^{\circ}$

The individual analyses show that the introduced initial imperfections can induce, during the initial phase of loading, small buckles located close to the saddle support. They may act as additional imperfections and may cause increasing of the limit force required to overcome them. The final shape of deformation of the perfect and imperfect structure is again similar (see Fig. 12 and Fig. 13).

\section{Conclusion}

The article was devoted to the theoretical description of carrying capacity of horizontal cylindrical shell on saddle supports. The computational models with embracing angles $60^{\circ}, 90^{\circ}$ and 


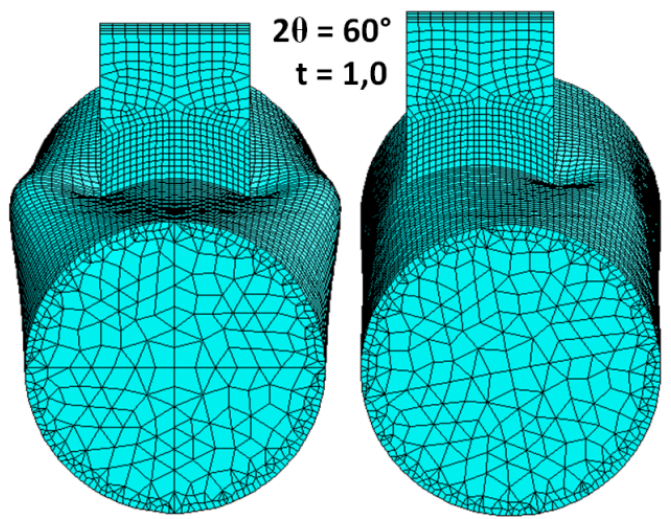

Fig. 12. Deformed perfect model at the beginning (left) and the end of loading (right), $t=1.0 \mathrm{~mm}, 2 \theta=60^{\circ}$

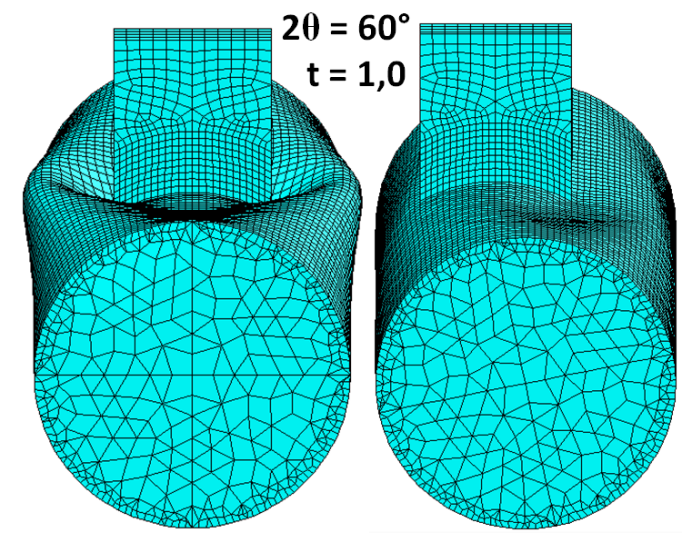

Fig. 13. Deformed imperfect model at the beginning (left) and the end of loading (right), $t=1.0 \mathrm{~mm}, 2 \theta=60^{\circ}$

$120^{\circ}$ were examined. Based on the evaluation of the first series of numerical analyses, it appears that the effect of initial shape imperfections on the loss of stability of laterally loaded cylindrical shells changes with the embracing angle $2 \theta$. The initial imperfections of the models with the largest angle $2 \theta=120^{\circ}$ led to a reduction of carrying capacity. Although carrying capacity of imperfect models with an embracing angle of $90^{\circ}$ was also mostly reduced, about one third of shells performed a slight reinforcing effect. Models with the embracing angle $2 \theta=60^{\circ}$ performed a reinforcement of the shell in almost all observed cases.

The results also show that the vast majority of examined cases lose stability at the first natural mode. This means that the cylindrical part of the model deviates from the vertical direction. The shell has a tendency to lay on one edge of the saddle. This is true regardless of the embracing angle and it is also a very important finding for the planned experimental verification. For example, Fig. 3 shows the load (equilibrium) curve where six gradual losses of stability occur during the loading of the shell. Each loss of stability causes decrease of carrying capacity and is accompanied by redistribution of deformation into new shape. However, the results of the analyses in the elastic area are not comparable to the experiments. The real experimental models lose stability in the elastic-plastic area. This is the aim of following research, which will include the influence of plasticity according to ECCS [2], by using $\chi$ (buckling resistance reduction factor for elastic-plastic effects).

Finally, it may be noted that the minimum value of the achieved reduction factor $\alpha_{\min }$ is approximately twice the value of the reduction factor $\alpha_{x}$ (for class B) provided by the ECCS [2] for axially compressed cylindrical shell. This conclusion is valid for all the embracing angles. Particular cases of these minimum values of reduction factor $\alpha_{\min }$ are shown in Fig. 5, Fig. 8 and Fig. 11

\section{References}

1 Bushnell D, Computerized buckling analysis of shells, Nijhoff; Dordrecht, 1985, ISBN 90-247-3099-6.

2 ECCS, Buckling of steel shells: European design recommendations, 5th ed., ECCS, 2008, ISBN 92-9147-000-92.

3 Eurocode 3: Design of steel structures - Part 1-6: Strength and stability of shell structures, 2007.

4 FEM Computer program COSMOS/M: Version 2.95, 2010.

5 Paščenko P, Spherical Partition of Road Tank Subjected to External Pressure, Scientific Papers of the University of Pardubice, Series B, The Jan Perner Transport Faculty, 2008(14), (2009), 81-92.

6 Paščenko P, Stejskal P, Road tank NKA 46.: Strength analysis - preparation of measurement in driving tests, Developmental report of AKMechanika, (2008).

7 Volmir AC, Ustojčivost' uprugich sistěm, Gosudarstvennoe izdastělstvo fiziko-matematiceskoj literatury; Moskva, 1963.

8 Voltr O, Paščenko P., Loss of stability of laterally loaded cylindrical shell with initial imperfection, TechMat 2012, In: Proceedings of the TechMat'12, Univerzita Pardubice; Pardubice, 2012, pp. 189-196 (cze).

9 Wunderlich W, Albertin U, Analysis and load carrying behavior of imperfection sensitive shells, International journal for numerical methods in engineering, 49, (2000), 255-273.

10 Wunderlich W, Deml M, Direct evaluation of the worst imperfection shape in shell buckling, Computer methods in applied mechanics and engineering, 149, (1997), 201-222, DOI 10.1016/S0045-7825(97)00055-8

11 Zienkiewicz OC, The Finite Element Method in Engineering Science, 2nd ed., McGRAW-HILL; London, 1971. 